\title{
Surprise Diagnosis of COVID-19 following Neuroimaging Evaluation for Unrelated Reasons during the Pandemic in Hot Spots
}

\author{
(D) Jain, (D) M. Young, (D) S. Dogra, (D) Hennedy, (D) V. Nguyen, and (D) E. Raz
}

\begin{abstract}
SUMMARY: During the height of the recent outbreak of coronavirus 19 (COVID-19) in New York City, almost all the hospital emergency departments were inundated with patients with COVID-19, who presented with typical fever, cough, and dyspnea. A small number of patients also presented with either unrelated conditions (such as trauma) or other emergencies, and some of which are now known to be associated with COVID-19 (such as stroke). We report such a scenario in 17 patients who were admitted and investigated with CT spine imaging and CT angiography for nonpulmonary reasons (trauma $=13$, stroke $=4$ ). Their initial work-up did not suggest COVID-19 as a diagnosis but showed unsuspected/incidental lung findings, which led to further investigations and a diagnosis of COVID-19.
\end{abstract}

D uring the height of the recent outbreak of coronavirus 19 (COVID-19) in New York City, one of the worst affected regions in the United States, almost all hospital emergency departments were inundated with patients with COVID-19, who presented with typical fever, cough, and dyspnea, though a small number of patients also presented with either unrelated conditions (eg, trauma) or other atypical emergencies, some of which are now known to be associated with COVID-19 (eg, stroke). ${ }^{1}$ We discuss 17 such patients who presented to the emergency department with nonpulmonary symptoms and did not raise the suspicion of COVID-19 at the time of admission. Initial nonpulmonary imaging in the emergency department led to the discovery of incidental lung findings that triggered further testing for Severe Acute Respiratory Syndrome coronavirus 2 (SARS-CoV2) with an eventual diagnosis of COVID-19.

\section{MATERIALS AND METHODS}

Four hundred seventy-three of a total of 3160 patients positive for COVID-19 who were admitted to NYU Langone Health, New York City, between March 1, 2020, and April 13, 2020, were investigated with various neuroimaging examinations (CT, MR imaging, and angiography), some of which included partial coverage of the bilateral lung fields. A confirmed case of COVID-19 was defined as a positive result on a real-time reverse

Received April 21, 2020; accepted after revision May 1.

From the Departments of Radiology (R.J., M.Y., S.D., H.K., V.N., E.R.) and Neurosurgery (R.J.), NYU Langone Health, New York, New York.

Please address correspondence to Rajan Jain, MD, Radiology and Neurosurgery, NYU Grossman School of Medicine, 660 First Ave, 2nd Floor, NY, NY 10016;

e-mail: rajan.jain@nyulangone.org; @CoolAsANeuroRad

- Indicates open access to non-subscribers at www.ajnr.org

http://dx.doi.org/10.3174/ajnr.A6608 transcriptase polymerase chain reaction assay of nasopharyngeal or oropharyngeal swab specimens. While reviewing these 473 patients, we identified a subgroup of 17 patients (range, 4493 years of age; mean age, 72 years; male/female ratio: 8:9) in whom COVID-19 as a diagnosis was not suspected at the time of admission and who underwent neuroimaging.

This study was approved by the NYU Grossman School of Medicine institutional review board, which granted both a waiver of informed consent and a waiver of the Health Insurance Portability and Accountability Act.

\section{RESULTS}

The reason for the imaging study in these 17 patients was trauma $(n=13)$ evaluated with spine CT (cervical spine $=11$, thoracic spine $=2$; with coverage of the posterior lungs) and acute stroke $(n=4)$ evaluated with CT angiography of the brain and neck (with coverage of the lung apices). Twelve of 13 patients with trauma had a history of a mechanical fall related to syncope or dizziness. These CT studies did not show any findings related to spine trauma or acute stroke but revealed suspicious lung findings with multifocal, peripheral, ground-glass opacities in both lungs (Figure). Lung findings were mentioned prospectively in the imaging reports as suspicious for pneumonia and viral etiology. All these patients underwent further laboratory investigations based on the surprising lung findings, and the diagnosis of COVID-19 was confirmed with a reverse transcriptase polymerase chain reaction assay of nasopharyngeal or oropharyngeal swab specimens, according to the World Health Organization interim guidance. ${ }^{2}$ Eight of 17 patients on further questioning after the COVID-19 diagnosis did confirm a history of cough in the days preceding presentation to the emergency department. Despite the initial admission not being related to pulmonary symptoms, 14 patients were eventually managed for varying 


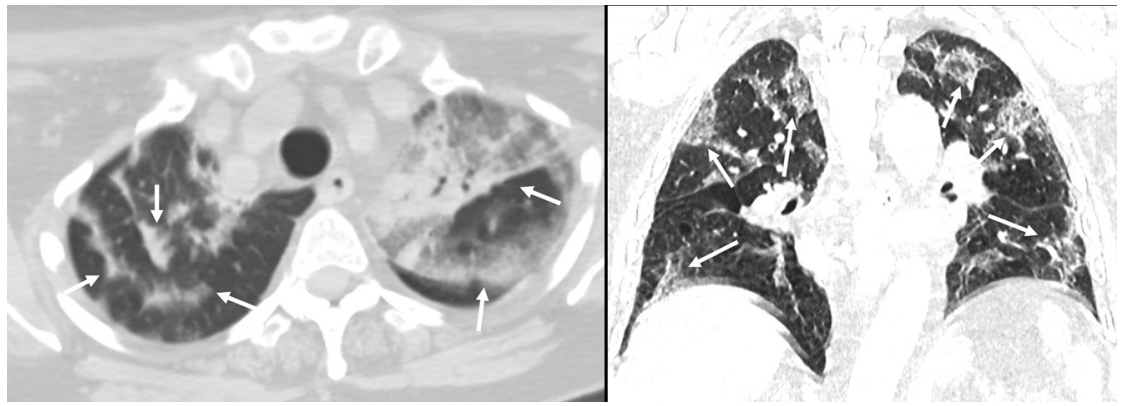

FIGURE. Axial and coronal CT images of 2 different patients, obtained from CTA of the neck (stroke evaluation) and CT of the thoracic spine (trauma evaluation), respectively, demonstrating typical multifocal, peripheral, ground-glass opacities in both lungs.

severity of respiratory symptoms and 6 patients had high oxygen requirements during their hospital stay. Three patients required a non-rebreather mask with an oxygen flow rate of $>15 \mathrm{~L} / \mathrm{min}$, and 3 patients needed endotracheal intubation and ventilator support. These patients required hospital admission for a mean of 7.29 days (range, 2-15 days), and 2 patients ultimately died due to COVID19-related complications (1- and 6- day hospital stays).

\section{DISCUSSION}

One of the major reasons for the very high community spread of SARS-CoV-2 (COVID-19) is that viral loads are being detected in asymptomatic individuals or mildly symptomatic patients. ${ }^{3,4}$ Fiftysix percent of asymptomatic residents of a skilled nursing home facility tested positive for COVID-19 in a recent study and likely contributed to disease transmission. ${ }^{4}$ High viral loads are almost always present soon after symptom onset, unlike SARS in 2002-2003, when viral shedding only happened after a patient was extremely symptomatic. ${ }^{3}$ Our study builds on this evidence by demonstrating that during the height of the COVID-19 pandemic in hot spots, there are many unsuspected and asymptomatic patients who are admitted to the emergency department and urgent care centers for either non-COVID-19-related reasons or atypical COVID-19 symptoms. These patients may neither be initially screened for COVID-19 nor isolated with precautions needed to curb the spread of the virus because the admission appears unrelated to COVID-19. Therefore, they may come in contact with multiple health care workers in the emergency department, including first responders or even various imaging/laboratory technologists, who may not have taken all the required precautions while handling or treating them.
Our case series will have a major impact on medical practice policies. For example, imaging/laboratory technologists or even receptionists at these clinics, laboratories, and imaging centers who will be coming in first contact with many of these otherwise asymptomatic patients must enforce better physical distancing and strict contact precautions and improved screening practices. This practice will be especially important as hospitals/clinics start to open up their facilities for outpatient work in the near future. All patients presenting at inpatient and outpatient imaging facilities at NYU Langone Health will now be screened for COVID-19-related symptoms and will need to wear masks regardless of their COVID-19 status, apart from similarly modified practice rules for radiology technologists and receptionists at the clinics and imaging centers.

\section{ACKNOWLEDGMENT}

We would like to acknowledge the NYU Family Connect Program as well as thousands of front-line health care workers who were an inspiration for this project.

Disclosures: Rajan Jain-UNRELATED: Board Membership: Nuevozen Corp; Consultancy: Cancer Panels Inc; Royalties: Thieme. Eytan Raz-UNRELATED: Expert Testimony: various law firms; Royalties: Springer; Travel/Accommodations/Meeting Expenses Unrelated to Activities Listed: MicroVention.

\section{REFERENCES}

1. Mao L, Jin H, Wang M, et al. Neurologic manifestations of hospitalized patients with coronavirus disease 2019 in Wuhan, China. JAMA Neurol 2020 Apr 10. [Epub ahead of print] CrossRef Medline

2. World Health Organization. Clinical management of severe acute respiratory infection when novel coronavirus $(\mathrm{nCoV})$ infection is suspected: interim guidance. March 13, 2020. https:/www.who.int/ publications-detail/clinical-management-of-severe-acute-respiratoryinfection-when-novel-coronavirus-(ncov)-infection-is-suspected. Accessed February 5, 2020

3. Zou L, Ruan F, Huang M, et al. SARS-CO-2 viral load in upper respiratory specimens of infected patients. N Engl J Med 2020;382:117779 CrossRef Medline

4. Arons MM, Hatfield KM, Reddy SC, et al. Presymptomatic SARSCoV-2 infections and transmission in a skilled nursing facility. N Engl J Med 2020 Apr 24. [Epub ahead of print] CrossRef Medline 